\title{
Utilización de nuevas materias primas y residuos industriales para mejorar las posibilidades de uso de los materiales cerámicos del área de Bailén (Jaén)
}

\author{
Use of new raw materials and industrial wastes to improve \\ the possibilities of using ceramic materials from Bailén \\ (Jaén, southern Spain)
}

\author{
R. J. Galán-Arboledas(*), A. Merino(*), $\underline{\text {. Bueno }}^{(*)}$
}

Recepción/Received: 17-VII-12

Aceptación/Accepted: 2-XI-12

Publicado online/Online publishing: 30-XI-12

\section{RESUMEN}

La materia prima empleada por la industria cerámica de Jaén consiste en mezclas de margas de la Depresión del Guadalquivir con arcillas rojas del Macizo Ibérico. La formulación de estas mezclas responde generalmente a la experiencia empírica desarrollada por esta industria durante generaciones. El presente trabajo evalúa las posibilidades de utilización de estas materias primas para fabricar nuevos productos cerámicos de construcción basándose en sus propiedades físico-químicas y analiza las limitaciones que presentan para la fabricación de productos de mayor valor añadido. Como alternativa para superar estas limitaciones se propone la mezcla con arcillas pirofilíticas de áreas geográficamente cercanas y con diferentes residuos industriales. Estos residuos son vidrio de pantalla descontaminado y tierras diatomeas impregnadas con aceites vegetales. El estudio de caracterización tecnológica muestra que las nuevas mezclas permiten el procesamiento de piezas cerámicas y que en ellas se mejoran o mantienen las propiedades estructurales y de aislamiento térmico.

Palabras clave: arcilla; cerámica estructural; valorización de residuos; aislamiento térmico.

\begin{abstract}
Raw materials used by the ceramic industry in Jaén (southern Spain) consist of mixtures of carbonated clays from the Guadalquivir Basin and red clays from the Iberian Massif. The mixtures formulation usually obeys empirical experience developed by this industry for many generations. This work, evaluates different possibilities of using these raw materials to manufacture new ceramic products on the basis of clay physical-chemical properties and analyzes limitations to produce high added value products. As an alternative to overcome these limitations, the mixture of these clays with raw materials from near regions (pyrophyllite clay) and with different industrial wastes is proposed. These wastes are screen glass from monitors and oil impregnated diatomaceous earth. The study of the technological properties shows that the new mixtures are suitable for the processing of ceramic products in which structural and thermal insulating properties are improved or kept.
\end{abstract}

Keywords: clay; structural ceramics; waste valuation; thermal insulation.

(*) Fundación Innovarcilla (Centro Tecnológico de la Cerámica de Andalucía). Bailén (Jaén, España).

Persona de contacto / Corresponding author. materiasprimas@innovarcilla.es 


\section{INTRODUCCIÓN}

En el "cluster" cerámico de Bailén (Jaén) se produce aproximadamente el $20 \%$ de la producción nacional de cerámica estructural para la construcción. Las materias primas utilizadas por esta industria consisten generalmente en mezclas de arcillas carbonatadas Neógenas de la Depresión del Guadalquivir con arcillas rojas procedentes del Trías que recubre el Macizo Ibérico. Las mezclas cerámicas propuestas con estas materias primas resultan, en general, adecuadas solamente para la fabricación de cerámica roja porosa (1-3), habiéndose llevado a cabo diferentes intentos para mejorar las prestaciones de estas mezclas mediante la adición de otras materias primas como caolín, feldespato o diatomita (1) u otros potenciales recursos mineros de la provincia de Jaén (4-6).

En este trabajo se proponen nuevas formulaciones para mejorar las prestaciones de estas mezclas cerámicas con objeto de obtener productos de mayor valor añadido o con un menor impacto ambiental. Por una parte, se han considerado los estudios que revelan cómo las materias primas ricas en pirofilita presentan buenas propiedades físico-químicas para ser utilizadas por la industria cerámica (7). En concreto, en el presente trabajo se ha utilizado una arcilla pirofilítica (arcilla Gris de Almuradiel) en explotación en un área geográficamente cercana a la provincia de Jaén (sur de la provincia de Ciudad Real) y con un destacable contenido de $\mathrm{Al}_{2} \mathrm{O}_{3}$. Por otra parte, existe una tendencia global en la industria de los materiales a considerar el reciclaje de residuos como una nueva fuente de materia prima. Así, la utilización de productos de construcción que incorporan material reciclado constituye uno de los criterios básicos que los diferentes métodos de diagnóstico utilizan para certificar una edificación como sostenible $(8,9)$.

En particular, la industria cerámica presenta procesos de fabricación que hacen especialmente viable la valorización de residuos, tanto si se trata de una valorización energética como de una valorización material de los propios residuos. Una revisión (10-12) de las principales experiencias de incorporación de residuos dentro de los materiales cerámicos permite distinguir dos grupos de residuos: de naturaleza orgánica o biomasa y de naturaleza inorgánica.

Los residuos de naturaleza orgánica aportan energía al liberar su poder calorífico durante la combustión que sufren en el proceso de cocción cerámico, lo que da lugar a una disminución de los gastos energéticos de fabricación al disminuir el uso de combustibles, aunque esto exige un mayor control de emisiones gaseosas. Dicha combustión proporciona además a los productos de arcilla cocida una microestructura más porosa, disminuyendo su densidad y mejorando previsiblemente su capacidad de aislamiento térmico (13-18).

\section{INTRODUCTION}

The ceramic cluster in Bailén (Jaén) produces around $20 \%$ of Spanish national production of structural ceramics for building. The raw materials used by this industry usually consist of mixtures of carbonated clays from the Neogene Guadalquivir Basin and Triassic red clays from the Iberian Massif. These clay mixtures are, in general, only adequate for the production of red porous ceramics for masonry (1-3). There have been some previous attempts in order to improve the performance of these mixtures through the addition of different kinds of raw materials, such as kaolin, feldspar or diatomite (1), or other potential mineral resources in Jaen province (4-6).

In this work, new formulations to improve the features of the traditional mixtures are proposed in order to get ceramic products with a higher added value or a lower environmental impact. On one hand, good physicalchemical properties of pyrophyllite clays have been considered to be used by the ceramic industry (7). In particular, in the present work, a pyrophyllite clay (Grey clay from Almuradiel) has been used due to its proximity (south of Ciudad Real province) to Jaen province and to its significant $\mathrm{Al}_{2} \mathrm{O}_{3}$ content. On the other hand, there is a global trend in the materials industry to consider the recycling of wastes as a new source of raw materials. In this way, the use of building materials incorporating recycled wastes constitutes one of the basic criterions that different assessment methods use to certify a building as sustainable $(8,9)$.

In particular, ceramic industry presents manufacture processes especially suitable for waste valuation, as much if it is an energy recovery as a material recovery of waste itself. $A$ review (10-12) of main experiences in waste valuation into ceramics allows identifying two groups of wastes: organic ones or biomass and those having an inorganic nature.

Organic wastes release their calorific value during the combustion produced in the firing process to manufacture ceramics, giving rise to lower energetic costs during manufacturing but to a stricter control of gas emissions. In addition, this combustion produces a porous microstructure in the fired ceramic products, diminishing their density and, likely, improving their ability for thermal insulation (13-18). 
Por otra parte, los residuos de naturaleza inorgánica formados principalmente por $\mathrm{SiO}_{2}-\mathrm{Al}_{2} \mathrm{O}_{3}-\mathrm{CaO}$, acompañados generalmente por otros óxidos metálicos minoritarios, presentan una composición similar a la de las materias primas cerámicas, por lo que estos residuos pueden pasar a formar parte de la matriz cerámica del producto y adquirir un carácter inerte debido a la formación de fases cristalinas y/o a una vitrificación durante el proceso de cocción. Así, es posible conseguir una amplia gama de productos que van desde los materiales vitrocerámicos a las cerámicas refractarias (1925). Esta valorización permite un ahorro de materias primas $y$, en algunas ocasiones, un menor consumo energético para obtener materiales con unas prestaciones optimizadas.

En el presente trabajo se exponen los primeros resultados de la utilización de dos residuos industriales de alto contenido en $\mathrm{SiO}_{2}$ : tierras diatomeas impregnadas de aceites vegetales y vidrio de pantalla descontaminado, dentro de las mezclas formadas únicamente por arcillas de Bailén o de aquellas que incorporan también la arcilla pirofilítica seleccionada. La caracterización preliminar realizada sobre estos materiales incluye la determinación de las principales propiedades tecnológicas, así como de la conductividad térmica, con objeto de establecer una primera viabilidad para la industrialización de nuevos materiales.

\section{PARTE EXPERIMENTAL}

\subsection{Materiales de partida}

Tradicionalmente la industria cerámica de Bailén ha utilizado cuatro tipos de arcillas para fabricar materiales cerámicos estructurales. Estas son las arcillas Blanca, Rubia y Negra, procedentes de la sedimentación marina que rellenó la cuenca del Guadalquivir durante el Neógeno y la arcilla Roja, que forma parte de los materiales del Trías que recubren el Macizo Ibérico. En el presente trabajo, como materias primas adicionales, se propone el uso de la arcilla Gris de Almuradiel, G, residuo de Tierras Diatomeas, D, procedentes de la filtración de aceites vegetales, y residuo de Vidrio de Pantalla, P. En la Tabla 1 se muestran las formulaciones tradicionalmente realizadas, únicamente con las arcillas de Bailén, para dos tipos de productos característicos: producto poroso para particiones interiores, generalmente revestidas con yeso o mortero (mezcla R) y una mezcla de mayor densidad generalmente destinada a la fabricación de productos de cara vista (Mezcla V). Las nuevas formulaciones con la arcilla Gris y los residuos propuestos, tomando como base las mezclas $\mathrm{R}$ y $\mathrm{V}$, también se muestran en la Tabla 1 .

\subsection{Técnicas experimentales}

La composición química de las arcillas y residuos de partida se ha determinado mediante fluorescencia de rayos $X$ (Philips PW 1404/10), mientras que la identificación semicuantitativa
On the other hand, inorganic wastes mostly formed by $\mathrm{SiO}_{2}-\mathrm{Al}_{2} \mathrm{O}_{3}-\mathrm{CaO}$ and, usually, some other minor metallic oxides, show a composition similar to that of ceramic raw materials. Therefore, these wastes can constitute the matrix of ceramic materials due to the formation of crystalline phases or to vitrification during the firing process and, thus, giving rise to inertization of the wastes. In this way, it is possible to get a wide range of ceramic products covering from glass-ceramics to refractory ceramics (19-25). Valuation of these inorganic wastes allows raw materials savings and, sometimes, a lower energy consumption to obtain optimized material properties.

In the present work, the first results of using two wastes with a high $\mathrm{SiO}_{2}$ content: oil impregnated diatomaceous earths and screen glass from monitors, are shown. The addition of these wastes was performed on those traditional mixtures containing only clays from Bailén and the new ones also containing the selected pyrophyllite clay. $A$ preliminary characterization of these materials comprises the determination of the main technological properties as well as thermal conductivity, in order to establish the suitability for industrialization of new materials.

\section{EXPERIMENTAL PART}

\subsection{Raw materials}

Ceramic industry in Bailén has traditionally used four types of clays to produce structural ceramics for building. These are the White, Yellow and Black clays from marine sediments that filled the Guadalquivir Basin during Neogene, and Red from the Triassic materials covering the Iberian Massif. In the present work, as additional raw materials, the use of Grey clay de Almuradiel, G, Diatomaceous Earth waste, $D$, from the filtration of vegetable oils, and Screen Glass waste, $P$, is proposed. Table 1 shows the formulation of traditional mixtures, using only clays from Bailén, for two characteristic ceramic products: porous bricks for internal masonry partitions covered with plaster or mortar (mixture $R$ ) and dense pieces usually aimed at the manufacturing of facing products (mixture V). New mixtures, including Grey clay and the selected wastes, have been formulated on the basis of $R$ and $V$ mixtures and are also shown in Table 1.

\subsection{Experimental techniques}

Chemical composition of clays and wastes has been determined by XRF (Philips PW 1404/10). Semiquantitative identification of mineral phases in clays, 
Tabla 1 / Table 1

Formulación de las mezclas. Los materiales porosos hacen referencia principalmente a materiales para revestir (R) mientras que los materiales densos se refieren generalmente a productos cara vista $(\mathrm{V})$.

Formulation of mixtures. Porous materials mainly refer to products to be coated $(R)$ while dense materials generally refer to facing products $(V)$.

\begin{tabular}{|c|c|c|c|c|c|c|c|c|}
\hline & & \multicolumn{4}{|c|}{$\begin{array}{c}\text { Arcillas de Bailén I } \\
\text { Clays from Bailén, Wt.\% }\end{array}$} & \multicolumn{3}{|c|}{$\begin{array}{l}\text { Nuevos recursos I } \\
\text { New resources Wt.\% }\end{array}$} \\
\hline & & $\begin{array}{c}\text { Blanca / } \\
\text { White }\end{array}$ & $\begin{array}{l}\text { Rubia I } \\
\text { Yellow }\end{array}$ & $\begin{array}{c}\text { Negra I } \\
\text { Black }\end{array}$ & $\begin{array}{l}\text { Roja I } \\
\text { Red }\end{array}$ & $\begin{array}{l}\text { Gris I } \\
\text { Grey }\end{array}$ & $D^{*}$ & $\mathbf{P}^{* *}$ \\
\hline \multirow{2}{*}{$\begin{array}{c}\text { Materiales porosos / } \\
\text { Porous Materials }\end{array}$} & $\mathrm{R}$ & 20 & 20 & 40 & 20 & - & - & - \\
\hline & $\mathrm{RP}$ & 18 & 18 & 36 & 18 & - & - & 10 \\
\hline \multirow{4}{*}{$\begin{array}{c}\text { Materiales densos / } \\
\text { Dense materials }\end{array}$} & VP & 22.5 & 22.5 & - & 45 & - & - & 10 \\
\hline & VG & - & - & - & 50 & 50 & - & - \\
\hline & VGP & - & - & - & 45 & 45 & - & 10 \\
\hline & VGD & - & - & - & 45 & 45 & 10 & - \\
\hline
\end{tabular}

*D: Tierras Diatomeas. / Diatomaceous Earth

${ }^{* *} \mathrm{P}$ : Vidrio de Pantalla. / Monitor Screen Glass.

de las fases minerales presentes en las arcillas de partida, tanto del polvo no orientado como de los agregados orientados, se ha realizado mediante DRX con un difractómetro Siemens D-500, empleando radiación de Cu-Ko y filtro de $\mathrm{Ni}$. La cuantificación de las muestras se ha realizado por el método de los poderes reflectantes relativos.

Para la fabricación de los materiales, todas las materias primas, arcillas y residuos se sometieron a molturación en molino de martillos con un tamiz de $3 \mathrm{~mm}$. A partir de las diferentes arcillas molturadas se han determinado los Límites de Atterberg (Límite Plástico, LP, y Límite Líquido, LL) y el Índice de Plasticidad (IP) mediante el método de Indentación (Hoytom, CM-C).

Tras el amasado de las diferentes mezclas con agua hasta una consistencia de $1,5 \mathrm{Kg} / \mathrm{cm}^{2}$, medida con penetrómetro de bolsillo (ST 207), se realizó el conformado de probetas con unas dimensiones de $12 \mathrm{~cm} \times 2,5 \mathrm{~cm} \times 1,6 \mathrm{~cm}$ mediante extrusión a vacío (Verdés, Monobloc 050-C/OR). Sobre las probetas en verde se realizó el estudio de las siguientes variables: agua de amasado, contracción lineal de secado, densidad y resistencia a flexión en tres puntos.

El comportamiento durante el tratamiento térmico se evaluó mediante ensayos termodilatométricos (Linseys, L76/1400D), utilizándose para los tratamientos de cocción 5 temperaturas máximas de trabajo: $850,900,950,1.000$ y $1.050{ }^{\circ} \mathrm{C}$ en un horno eléctrico de laboratorio (Kittec CB, CBN-50), manteniendo las piezas durante 3 horas a dichas temperaturas. Se han determinado mediante DRX (Phillips $X$ Pert PRO MPD) las fases mineralógicas presentes en la mezcla referencia $\mathrm{R}$ tratada a $900{ }^{\circ} \mathrm{C}$, utilizándose el método Rietveld para cuantificar la contribución de cada una de las fases cristalinas y del material no difractante al total de la muestra. on the bulk samples and oriented aggregates, was performed by XRD (Siemens D-500, Ni-filtered and with Cu-Ko radiation). Quantification of clay mineral components was obtained from the method of the reflectant powers.

In order to produce test specimens, all raw materials (clays and wastes) were dry ground by hammer mill (sieve $3 \mathrm{~mm}$ ). The ground powder was used to determine Atterberg Limits (Plastic Limit, $L P$, and Liquid Limit, $L L)$ and Plascticity Index (IP) by the indentation method (Hoytom, CM-C).

Mixing of powder and water was performed until achieving a consistency of $1.5 \mathrm{Kg} / \mathrm{cm}^{2}$ (measurement performed with a manual penetrometer, ST 207). Shaping of bars $(12 \mathrm{~cm} \times 2.5 \mathrm{~cm} \times 1.6 \mathrm{~cm})$ was carried out through vacuum extrusion (Verdés, Monobloc 050-C/OR). Unfired specimens were characterized to determine the following properties: working moisture, drying shrinkage, bulk density and bending strength.

Materials behaviour during the thermal treatment was evaluated by dilatometric tests (Linseys, L76/1400D). Firing was performed out in electric chamber kiln Kittec (CB, CBN-50) at five maximum temperatures: 850, 900, 950, 1000 and $1050^{\circ} \mathrm{C}, 3 \mathrm{~h}$ dwell. Mineral phases in reference material $R$, fired at $900{ }^{\circ} \mathrm{C}$, were determined by $X R D$ (Phillips X Pert PRO MPD), using Rietveld method to quantify the contribution of both crystalline phases and non-difractant material 
Sobre los materiales cocidos se determinaron las siguientes propiedades: densidad aparente, resistencia a flexión, contracción lineal y absorción de agua determinada según la norma UNE-EN ISO 10545-3. Los ensayos de resistencia a flexión en tres puntos (Hoytom, CM-C, 100 mm de distancia entre apoyos), se realizaron con una velocidad de desplazamiento de $10 \mathrm{~mm} / \mathrm{min}$ en los materiales en verde y de $5 \mathrm{~mm} / \mathrm{min}$ en los materiales cocidos. Adicionalmente se fabricaron losetas de dimensiones $10 \mathrm{~cm} \times 8 \mathrm{~cm}$ $x 1,1 \mathrm{~cm}$, a partir de las cuales se mecanizaron probetas para las medidas de aislamiento térmico. En concreto, la conductividad térmica se determinó a partir de una adaptación de la técnica del hilo caliente (Linseis, THB) sobre probetas rectangulares de $8 \mathrm{~cm} \times 4 \mathrm{~cm}$, con un espesor de $8 \mathrm{~mm}$.

\section{RESULTADOS Y DISCUSIÓN}

\subsection{Caracterización de las materias primas y formulación de nuevas mezclas}

Las arcillas neógenas son los materiales más extensamente usados por la industria cerámica de la región (1-2). Esta formación está compuesta por las arcillas Blanca, Rubia y Negra que, según los resultados de los análisis de DRX (Tabla 2), se caracterizan por ser ricas en cuarzo y calcita, predominando en ellas filosilicatos del tipo illita y esmectita, que otorgan a dichas arcillas una plasticidad mediaalta, pudiendo, por lo tanto, presentar algunos problemas de secado. Por el contrario, la arcilla Roja procedente de la Cobertura Tabular del Trías contiene dolomita y no se detecta calcita, siendo el filosilicato predominante la illita con algo de caolinita, lo que le confiere baja plasticidad y excelentes condiciones de secado. El contenido de feldespatos en las arcillas estudiadas no supera en ninguno de los casos el $6 \%$.
Fired specimens were characterized to determine the foIlowing: bulk density, bending strength, firing shrinkage and water absorption according to standard test UNEEN ISO 10545-3. Three point bending strength tests (Hoytom, CM-C, $100 \mathrm{~mm}$ span) were carried out using a displacement rate of $10 \mathrm{~mm} / \mathrm{min}$ and $5 \mathrm{~mm} / \mathrm{min}$ for unfired and fired specimens, respectively. In addition, plates $(10 \mathrm{~cm} \times 8 \mathrm{~cm} \times 1.1 \mathrm{~cm})$ were also obtained for thermal insulation characterization. In particular, thermal conductivity was determined by an adaptation of the hot wire technique (Linseis, THB) on specimens $8 \mathrm{~mm}$ thick and rectangular faces $(8 \mathrm{~cm} \times 4 \mathrm{~cm})$.

\section{RESULTS AND DISCUSSION}

\subsection{Raw materials characterization and formulation of new mixtures}

Neogene clays are the raw materials more widely used by the regional ceramic industry (1-2). This geological formation is composed of White, Yellow and Black clays that, according to DRX analysis (Table 2), are characterized by high carbonates and quartz contents. Predominant phyllosilicates in these clays are illite and smectite, giving rise to medium-high plasticity and, therefore, to some drying problems. By contrast, the Red Triassic clay contains only some dolomite whereas calcite is non detected (Table 2). The predominant phyllosilicate is illite, and kaolinite in a minor extension, thus providing excellent drying properties and low plasticity. Feldspars content in all the clays is less than 6 wt\%.

Tabla 2 / Table 2

Análisis mineralógico (\% en peso) y plasticidad de las arcillas y mezclas de arcillas tradicionales. Mineralogical composition (wt.\%) and plasticity of clays and traditional clay mixtures.

\begin{tabular}{|c|c|c|c|c|c|c|c|c|c|c|c|c|}
\hline & \multirow{2}{*}{$\mathbf{Q}$} & \multirow{2}{*}{ Fd } & \multirow{2}{*}{ C } & \multirow{2}{*}{ D } & \multirow{2}{*}{ FL } & \multicolumn{5}{|c|}{$\begin{array}{l}\text { Composición relativa de FL / } \\
\text { Relative FL composition }\end{array}$} & \multicolumn{2}{|c|}{$\begin{array}{c}\text { Plasticidad / } \\
\text { Plasticity, } \% \mathrm{H}_{2} \mathrm{O}\end{array}$} \\
\hline & & & & & & Es & III & $\mathbf{K}$ & CL & $\mathbf{P}$ & I.P & L.P \\
\hline Blanca / White & 28 & 6 & 25 & 8 & 33 & 68 & 32 & - & - & - & 29.9 & 15.5 \\
\hline Rubia / Yellow & 40 & - & 15 & - & 45 & 40 & 60 & - & $\mathrm{Tr}$ & - & 21.7 & 11.1 \\
\hline Negra / Black & 43 & 3 & 18 & 4 & 32 & 32 & 58 & 10 & - & - & 20.2 & 18.6 \\
\hline Roja / Red & 32 & 5 & - & 10 & 53 & - & 66 & 33 & $\mathrm{Tr}$ & - & 11.5 & 14.1 \\
\hline Gris / Grey & 5 & $\mathrm{Tr}$ & - & - & 93 & - & 30 & 19 & - & 51 & 26.2 & 20.4 \\
\hline $\mathrm{R}$ & 37 & $\mathrm{Tr}$ & 15 & 6 & 39 & 34 & 55 & 11 & $\mathrm{Tr}$ & - & 21.0 & 16.0 \\
\hline $\mathrm{V}$ & 33 & 4 & 10 & 7 & 46 & 27 & 56 & 17 & $\mathrm{Tr}$ & - & 19.0 & 14.0 \\
\hline
\end{tabular}

Q: Cuarzo / Quartz, Fd: Feldespatos / Feldspars, C: Calcita / Calcite, D: Dolomita / Dolomite, FL: Filosilicatos / Phyllosilicates,

Es: Esmectita / Esmectite, III: Illita / Illite, K: Caolinita / Kaolinite, Cl: Clorita / Chlorite, P: Pirofilita / Pyrofillyte. Tr: Trazas / Trace (1-3\%).

I.P: Índice de Plasticidad / Plasticity Index, L.P: Límite Plástico / Plastic Limit. 
La determinación de los límites de plasticidad de Atterberg (Tabla 2) verifica los resultados previstos a partir del análisis mineralógico, revelando, tal y como han puesto de manifiesto los estudios anteriores (1-3), que las arcillas Blanca y Roja no presentan aplicación cerámica por sí solas, ya que los valores de plasticidad de estas arcillas se encuentran fuera de los límites óptimos recomendados para el conformado mediante extrusión: $15-25 \% \mathrm{H}_{2} \mathrm{O}(1,4-5)$.

Según el análisis químico, Tabla 3, y de acuerdo con la proporción de carbonato cálcico, el contenido en $\mathrm{CaO}$ de las arcillas Negra, Rubia y Roja es inferior al contenido de la arcilla Blanca $(\approx 17 \%)$. La disminución del contenido de calcio en las arcillas se correlaciona con un aumento del contenido de $\mathrm{SiO}_{2}$ y $\mathrm{Al}_{2} \mathrm{O}_{3}$, alcanzando valores de casi un $60 \%$ de $\mathrm{SiO}_{2}$ y un $17 \%$ de $\mathrm{Al}_{2} \mathrm{O}_{3}$ en la arcilla Roja. Además, el mayor contenido de $\mathrm{K}_{2} \mathrm{O}$ de las arcillas Negra, Rubia y Roja refleja el carácter predominantemente illítico de estas arcillas respecto al carácter esmectítico de la arcilla Blanca. El contenido en Fe de la arcilla Roja (7\%) es significativamente superior al de las arcillas neógenas, con valores del $3-4 \%$.

Por lo que respecta a las mezclas de trabajo habituales ( $R$ y $V$ ) descritas en la Tabla 1, y que han sido utilizadas como materiales de referencia en el presente trabajo, la incorporación de las arcillas Blanca y Roja permite modificar el comportamiento de las arcillas Rubia y Negra. En concreto, la arcilla Blanca, rica en esmectitas y carbonatos y con un Índice de Plasticidad en torno al 30\% $\mathrm{H}_{2} \mathrm{O}$, se puede adicionar para proporcionar resistencia a las piezas en verde, pero solamente en pequeñas cantidades si no se quieren originar problemas de secado. Por el contrario, la arcilla Roja, sin carbonatos y con mayor contenido de alúmina, no presenta plasticidad suficiente para el conformado de piezas en verde con una resistencia adecuada. Debido a las proporciones utilizadas, las mezclas tradicionales, R y V (Tabla 1), poseen una plasticidad adecuada para el conformado (Tabla 2) y presentan un contenido medio-alto de carbonatos ( $\approx 20 \%$, Tabla 2 ) y una relación cuarzo / filosilicatos que las hace especialmente viables para la fabricación de ladrillos y tejas, tal y como ha sido puesto de manifiesto en $(1,2)$ basándose en los diagramas de Fiori y col. (26).

En concreto, una representación de la composición química de arcillas y mezclas (Tabla 3) en uno de los diagramas ternarios de Fiori y col. (Figura 1) permite evaluar la idoneidad de estas mezclas para la fabricación de otros tipos de productos, principalmente cerámicas para revestimiento (26). Dicho diagrama (Figura 1) confirma que las arcillas de Bailén y sus mezclas ( $\mathrm{V}$ y $\mathrm{V}$ ) o bien no tienen utilidad por sí solas o son aptas principalmente para la fabricación de revestimientos porosos, siendo la arcilla Roja la única que presenta viabilidad para la obtención de gres rojo.
The determination of Atterberg Limits (Table 2) verifies the results anticipated by mineralogical analysis. These results reveal, as previously described (1-3), that White and Red clays can not be used on their own for ceramics production since their plasticity values are out of the optimum recommended range for extrusion shaping: 15$25 \% \mathrm{H}_{2} \mathrm{O}(1,4-5)$.

According to chemical analysis, Table 3, and in agreement to calcium carbonate content (Table 2), CaO contents of Black, Yellow and Red clays are lower than content in White clay ( $\approx 17 \%)$. The decrease of calcium contents is correlated to an increase of $\mathrm{SiO}_{2}$ and $\mathrm{Al}_{2} \mathrm{O}_{3}$ contents, reaching values of $60 \% \mathrm{SiO}_{2}$ and $17 \% \mathrm{Al}_{2} \mathrm{O}_{3}$ in Red clay. In addition, the higher $\mathrm{K}_{2} \mathrm{O}$ contents in Black, Yellow and Red clays reflect the mainly illitic composition of these clays in relation to the smectitic composition of White clay. Iron content in Red clay (7\%) is significantly higher than in Neogene clays (3-4\%).

Regarding traditional mixtures ( $R$ and $V$ ) described in Table 1 , and used as reference materials in the present work, the addition of White and Red clays allows modifying the technological behaviour of Yellow and Black clays. In particular, White clay, with high smectite and carbonate contents and a Plasticity Index around 30\% $\mathrm{H}_{2} \mathrm{O}$, can be added to provide higher mechanical strength in unfired pieces bus just in small quantities to avoid drying problems. On the contrary, Red clay, low-carbonated and with higher alumina content, does not have sufficient plasticity for the shaping of resistant unfired pieces. Due to the proportions of clays used, traditional mixtures $R$ and $V$ (Table 1) have appropriate plasticity for shaping (Table 2) and present a medium-high carbonate content ( $\approx 20 \%$, Table 2) and a quartz / phyllosilicates ratio especially practical for the manufacturing of bricks and roof-tiles, as has been reviewed in $(1,2)$ on the basis of Fiori et al. charts (26).

In particular, a representation of chemical composition of clays and their mixtures (Table 3) in one of the triangular charts by Fiori et al. (Figure 1) allows evaluating the suitability of these mixtures for the manufacturing a different kind of products, mainly ceramics for coverings (26). The chart (Figure 1) confirms that clays from Bailén and their mixtures ( $R$ and $V$ ) do not have application on their own or that they are mainly suitable for the manufacturing of porous bodies, being Red clay the only one that show feasibility for the production of red stoneware. 


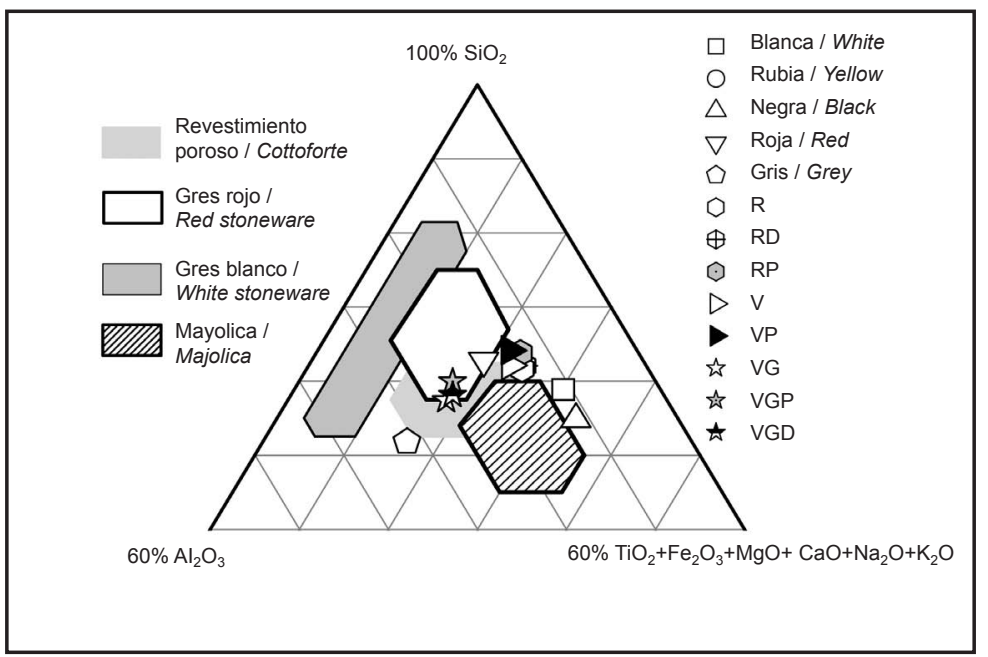

Figura 1. Representación de la composición química de las materias primas estudiadas en el diagrama de Fiori y col. (26). Existe un solapamiento de las composiciones de la arcilla Rubia y la mezcla RD.

Figure 1. Representation of chemical composition of studied raw materials in the chart of Fiori et al. (26). There is an overlapping of compositions corresponding to Rubia clay and RD mixture.

Tabla 3 / Table 3

Análisis químico (\% en peso) de las materias primas: arcillas, residuos y mezclas estudiadas. Chemical composition (wt.\%) of raw materials: clays, wastes and mixtures.

\begin{tabular}{|c|c|c|c|c|c|c|c|c|c|c|c|c|}
\hline & & $\mathrm{SiO}_{2}$ & $\mathrm{Al}_{2} \mathrm{O}_{3}$ & $\mathrm{Fe}_{2} \mathrm{O}_{3}$ & MnO & MgO & $\mathrm{CaO}$ & $\mathrm{Na}_{2} \mathrm{O}$ & $\mathrm{K}_{2} \mathrm{O}$ & $\mathrm{TiO}_{2}$ & $\mathrm{P}_{2} \mathrm{O} 5$ & $\begin{array}{l}\text { P.F.II I } \\
\text { LOI }\end{array}$ \\
\hline \multirow{4}{*}{$\begin{array}{c}\text { Arcillas } \\
\text { tradicionales / } \\
\text { Traditional } \\
\text { clays }\end{array}$} & Blanca / White & 47.5 & 8.8 & 3.3 & 0.0 & 1.7 & 16.6 & 0.4 & 1.9 & 0.5 & 0.1 & 17.7 \\
\hline & Rubia / Yellow & 56.6 & 12.1 & 4.2 & 0.0 & 2.2 & 9.1 & 1.7 & 3.1 & 0.0 & 0.0 & 10.8 \\
\hline & Negra / Black & 54.2 & 12.3 & 4.3 & 0.1 & 2.1 & 10.4 & 1.4 & 2.9 & 0.7 & 0.1 & 12.2 \\
\hline & Roja / Red & 58.5 & 16.7 & 7.0 & 0.1 & 2.8 & 2.2 & 0.2 & 5.0 & 0.8 & 0.2 & 6.7 \\
\hline \multirow{3}{*}{$\begin{array}{l}\text { Recurso / } \\
\text { Resource }\end{array}$} & Gris, G & 46.3 & 28.6 & 9.3 & 0.0 & 0.4 & 0.5 & 0.5 & 2.8 & 1.0 & 0.1 & 9.8 \\
\hline & Diatom., D & 31.8 & 1.7 & 0.6 & 0.0 & 8.7 & 0.3 & 1.1 & 0.4 & 0.0 & 0.8 & 53.1 \\
\hline & Vidrio, $P$ & 80.2 & 6.3 & 0.7 & 1.0 & 1.2 & 1.2 & 9.0 & 0.2 & 0.4 & 0.2 & 0.1 \\
\hline \multirow{8}{*}{$\begin{array}{l}\text { Mezclas I } \\
\text { Mixtures }\end{array}$} & $\mathrm{R}$ & 54.2 & 12.4 & 4.6 & 0.1 & 2.2 & 9.7 & 1.0 & 3.2 & 0.5 & 0.1 & 11.9 \\
\hline & $\mathrm{RD}$ & 52.5 & 11.6 & 4.3 & 0.1 & 2.7 & 9.0 & 1.0 & 3.0 & 0.5 & 0.2 & 15.0 \\
\hline & $\mathrm{RP}$ & 56.8 & 11.8 & 4.2 & 0.1 & 2.1 & 8.9 & 1.8 & 2.9 & 0.5 & 0.1 & 10.7 \\
\hline & $\mathrm{V}$ & 55.3 & 13.6 & 5.4 & 0.1 & 2.4 & 7.5 & 0.6 & 3.8 & 0.5 & 0.1 & 10.5 \\
\hline & VP & 57.8 & 12.8 & 4.9 & 0.1 & 2.3 & 6.9 & 1.5 & 3.4 & 0.5 & 0.1 & 9.4 \\
\hline & VG & 52.4 & 22.7 & 8.2 & 0.1 & 1.6 & 1.4 & 0.4 & 3.9 & 0.9 & 0.2 & 8.3 \\
\hline & VGP & 55.2 & 21.0 & 7.4 & 0.1 & 1.6 & 1.3 & 1.2 & 3.5 & 0.9 & 0.2 & 7.4 \\
\hline & VGD & 50.3 & 20.6 & 7.4 & 0.0 & 2.3 & 1.2 & 0.4 & 3.6 & 0.8 & 0.2 & 12.7 \\
\hline
\end{tabular}

* P.F. Pérdidas por calcinación / L.O.I Loss on ignition

Esto es debido, fundamentalmente, a un exceso de $\mathrm{CaO}$ en las arcillas neógenas, por lo que es de interés disminuir el contenido de carbonatos de las mezclas mediante un aumento del contenido de $\mathrm{SiO}_{2}$ y/o $\mathrm{Al}_{2} \mathrm{O}_{3}$. Para ello, en el presente trabajo se propone la utilización de los siguientes recursos: arcilla Gris de Almuradiel, residuo de Vidrio de Pantalla descontaminado y residuo de Tierras Diatomeas utilizadas para la filtración de aceites vegetales.

La característica principal de la arcilla Gris, en comparación con las arcillas utilizadas tradicionalmente por la industria ladrillera de Bailén, es la presencia de pirofilita como filosilicato predominante (Tabla 2) y un mayor contenido de $\mathrm{Al}_{2} \mathrm{O}_{3}(29 \%$, Tabla 3). Esta arcilla presenta una plasticidad
This is mainly due to an excess of CaO in Neogene clay and, therefore, it is of great interest to reduce carbonates contents in the mixtures through the increase of $\mathrm{SiO}_{2}$ and/or $\mathrm{Al}_{2} \mathrm{O}_{3}$. To do this, the present work proposes the use of the following resources: Grey clay from Almuradiel, Screen Glass waste and Diatomaceous Earths from filtration of vegetable oils.

The main feature of Grey clay, compared with clays used traditionally by the brick industry in Bailén, is the presence of pyrophyllite as predominant phyllosilicate (Table 2) and a higher $\mathrm{Al}_{2} \mathrm{O}_{3}$ content (29\%, Table 3). This clay shows high plasticity and does not contain carbonates 
elevada y no contiene carbonatos ni esmectitas, por lo que es una candidata idónea para mezclar con la arcilla Roja de Bailén, lo que daría lugar a una mezcla con bajo contenido de carbonatos y con una plasticidad adecuada para el conformado de piezas cerámicas.

En cuanto a los residuos industriales, hay que destacar el alto contenido de $\mathrm{SiO}_{2}(80,2 \%)$ y $\mathrm{Na}_{2} \mathrm{O}(9 \%)$ del Vidrio de Pantalla (Tabla 3). Igualmente, en las Tierras Diatomeas, una vez descontadas las pérdidas por calcinación que supone el aceite vegetal retenido en ellas (53\% en peso, Tabla 3), destaca el contenido en $\mathrm{SiO}_{2}$ (32\% del total y $68 \%$ de la fracción inorgánica, Tabla 3). Por lo tanto, ambos residuos se pueden considerar como importantes fuentes de $\mathrm{SiO}_{2}$ y sales fundentes.

Teniendo en cuenta las características de las arcillas tradicionales de Bailén y de los nuevos recursos planteados (arcilla Gris y residuos de alto contenido en $\mathrm{SiO}_{2}$ ), se han propuesto una serie de nuevas formulaciones encaminadas a mejorar las prestaciones y posibilidades de uso de las mezclas tipo $\mathrm{R}$ y $\mathrm{V}$ (Tabla 1 ), normalmente utilizadas para la fabricación de ladrillos. Estas nuevas mezclas se recogen en la Tabla 1: RP, RD, VG, VP, VGP y VGD (donde G: Gris de Almuradiel, D: Tierras Diatomeas, P: Vidrio de Pantalla). En la mezcla $R$, con una plasticidad óptima para el proceso de extrusión, la adición de un $10 \%$ en peso de Vidrio de Pantalla y Tierras Diatomeas (mezclas RP y RD, Tabla 1), como recursos de alto contenido en $\mathrm{SiO}_{2}$, permite reducir ligeramente el contenido de carbonatos de la mezcla sin reducir excesivamente $(\approx 10 \%)$ la plasticidad de la misma. En el caso de la mezcla $V$, la sustitución de las arcillas neógenas (Blanca y Rubia) por la arcilla Gris permite, como se ha señalado anteriormente, obtener una mezcla (VG) de bajo contenido en carbonatos, manteniendo una plasticidad suficiente para el conformado (IP $\approx 18,9 \% \mathrm{H}_{2} \mathrm{O}$ ). La adición de un $10 \%$ de los residuos seleccionados sobre las mezclas V y VG (Mezclas VP, VGP y VGD, Tabla 1) permite incrementar el contenido de $\mathrm{SiO}_{2}$ hacia la zona idónea de fabricación de gres en el diagrama de la Figura 1. De hecho, la representación de la composición química de las nuevas mezclas propuestas en dicho diagrama muestra que las mezclas VG, VGD y VGP son candidatas para fabricar gres rojo, mientras que las mezclas VP, RD y RP son aptas únicamente para revestimientos porosos, al igual que sus mezclas de partida. Por lo tanto, la dosificación de residuos propuesta en estas últimas mezclas no parece suficiente para mejorar las prestaciones del producto final. Una verificación de esta predicción empírica requiere de la caracterización tecnológica de los materiales que se muestra en el apartado 3.2.

\subsection{Caracterización tecnológica de los materiales}

En la Tabla 4 se muestran los resultados de la caracterización de los materiales en verde obtenidos a partir de las mezclas propuestas. Los valores determinados indican or smectites and, therefore, is a suitable candidate for mixing with Red clay from Bailén, giving rise to a mixture of low carbonates content and adequate plasticity for the shaping of ceramic products.

Concerning the wastes, it is remarkable the high content of $\mathrm{SiO}_{2}(80.2 \%)$ and $\mathrm{Na}_{2} \mathrm{O}$ (9\%) of the Glass Screen (Table 3). Similarly, in the Diatomaceous Earth, after deduction of the loss on ignition which is vegetable oil retained therein (53 wt\%, Table 3), highlights the content on $\mathrm{SiO}_{2}$ (32\% of the total and $68 \%$ of the inorganic fraction, Table 3). Therefore, both wastes can be considered as important sources of $\mathrm{SiO}_{2}$ and melting salts.

Taking into account the characteristics of traditional clays from Bailén and of the new resources proposed (Grey clay and wastes with high $\mathrm{SiO}_{2}$ contents) a number of new formulations have been proposed in order to improve performance and usability of mixtures $R$ and $V$ (Table 1) normally used for the manufacture of bricks. These new mixtures are shown in Table 1: $R P, R D, V G, V P, V G P$ and VGD (where G: Grey clay from Almuradiel, D: Diatomaceous Earth, $P$ : Screen Glass). In mixture $R$, with an optimal plasticity for the extrusion process, the addition of 10 wt.\% of Glass Screen and Diatomaceous Earths (mixtures $R P$ and $R D$, Table 1), as resources with high $\mathrm{SiO}_{2}$ content, reduces slightly carbonate content of the mixture without unduly reducing $(\approx 10 \%)$ the plasticity thereof. In the case of mixture $V$, replacement of $\mathrm{Neo}$ gene clays (White and Yellow) by the Grey clay allows, as stated above, to obtain a mixture (VG) of low content of carbonates, maintaining enough plasticity for shaping $\left(I P \approx 18.9 \% \mathrm{H}_{2} \mathrm{O}\right.$ ). The addition of $10 \%$ of the selected wastes on the mixtures $V$ and VG (mixtures $V P, V G P$ and $V G D$, Table 1) allows to increase the content of $\mathrm{SiO}_{2}$ to the area suitable for manufacturing stoneware tiles in the diagram of Figure 1 In fact, the representation of the chemical composition of the novel mixtures proposed in the diagram shows that mixtures VG, VGD and VGP are candidates for manufacturing red stoneware, while mixtures $V P, R D$ and $R P$ are only suitable for porous coverings, similarly to the starting mixtures. Therefore, the dosage of the proposed wastes in the latest mixtures may not be sufficient to improve the performance of the final product. A verification of this empirical prediction requires the technological characterization of the materials shown in paragraph 3.2.

\subsection{Technological properties}

Table 4 shows the results of the characterization of the unfired materials obtained from the mixtures proposed. Determined values indicate that both the use of wastes 
Tabla 4 / Table 4

Propiedades tecnológicas de los materiales en seco. Technological properties of unfired materials.

\begin{tabular}{|c|c|c|c|c|c|c|c|c|}
\hline & $\mathbf{R}$ & $\mathbf{R D}$ & $\mathbf{R P}$ & $\mathbf{V}$ & $\mathbf{V P}$ & $\mathbf{V G}$ & VGP & VGD \\
\hline $\begin{array}{c}\mathrm{AA} \\
(\%)\end{array}$ & $22.1 \pm 0.1$ & $24.7 \pm 0.2$ & $19.9 \pm 0.2$ & $22.9 \pm 0.1$ & $20.6 \pm 0.1$ & $21.7 \pm 0.1$ & $20.3 \pm 0.3$ & $21.3 \pm 0.2$ \\
\hline $\begin{array}{c}\mathrm{CL} \\
(\%)\end{array}$ & $5.8 \pm 0.2$ & $5.4 \pm 0.1$ & $5.0 \pm 0.1$ & $5.7 \pm 0.1$ & $4.9 \pm 0.1$ & $4.7 \pm 0.2$ & $4.2 \pm 0.2$ & $3.4 \pm 0.1$ \\
\hline $\mathrm{IN}$ & $0.72 \pm 0.04$ & $0.61 \pm 0.02$ & $0.64 \pm 0.03$ & $0.68 \pm 0.02$ & $0.60 \pm 0.02$ & $0.51 \pm 0.03$ & $0.46 \pm 0.04$ & $0.37 \pm 0.01$ \\
\hline $\begin{array}{c}\mathrm{DA} \\
\left(\mathrm{g} / \mathrm{cm}^{3}\right)\end{array}$ & $1.96 \pm 0.01$ & $1.81 \pm 0.01$ & $2.04 \pm 0.01$ & $1.98 \pm 0.01$ & $2.06 \pm 0.01$ & $2.02 \pm 0.01$ & $2.05 \pm 0.01$ & $1.86 \pm 0.01$ \\
\hline $\begin{array}{c}\mathrm{RM} \\
\left(\mathrm{Kg} / \mathrm{cm}^{2}\right)\end{array}$ & $70 \pm 4$ & $67 \pm 6$ & $72 \pm 3$ & $67 \pm 4$ & $64 \pm 5$ & $40 \pm 2$ & $36 \pm 2$ & $60 \pm 3$ \\
\hline
\end{tabular}

A.A.: Agua de amasado / Working moisture; C.L.: Contracción lineal / Linear Shrinkage; I.N.: Índice de Nosova / Nosova Index; D.A: Densidad aparente en seco / Dry Bulk Density; RM: Resistencia mecánica / Bending Strength.

que tanto la utilización de los dos residuos como de la arcilla Gris mejora notablemente el comportamiento de las mezclas de referencia $\mathrm{R}$ y $\mathrm{V}$ durante el proceso de secado, como demuestra la disminución de la contracción lineal y del índice de Nosova (Tabla 4). La utilización de Tierras Diatomeas provoca un descenso de la densidad aparente debido, principalmente, al elevado contenido de materia orgánica de este residuo (LOI $\approx 50 \%$, Tabla 3). Sin embargo, este descenso de densidad no se traduce en una pérdida de resistencia mecánica, lo que puede ser debido, en parte, al efecto plastificante del aceite vegetal impregnado en dichas tierras filtrantes (27). La introducción en las pastas tradicionales ( $R$ y $V$ ) de Vidrio de Pantalla provoca un aumento de densidad debido a la mayor densidad del propio residuo. Las resistencias mecánicas de las probetas en verde se encuentran por encima de $35 \mathrm{Kg} / \mathrm{cm}^{2}$ en todos los casos, por lo que, a priori, son mezclas aptas para su uso a nivel industrial, permitiendo la manipulación de las piezas durante su carga en el horno.

El comportamiento de las arcillas y las mezclas propuestas durante los ensayos de sinterización dinámica se muestra en la Figura 2. En ella se aprecia como la arcilla Blanca, de naturaleza esmectítica y con mayor contenido de carbonatos, comienza a sinterizar antes que las arcillas Rubia y Negra, de naturaleza illítica (Figura 2a). Debido a los altos contenidos de carbonatos de estas tres arcillas (Tabla 2) se aprecia como a partir de $900-950^{\circ} \mathrm{C}$ comienzan a formarse fases cálcicas cristalinas (Figura 2a) que mantiene en estos materiales un carácter refractario (28). Por el contrario, la ausencia de carbonatos de las arcillas Roja y Gris, y su carácter fundente (Figura 2a), hacen de ellas materias interesantes para la obtención de productos gresificados o clinker de mayor valor añadido. El mayor contenido de arcillas neógenas, especialmente Rubia y Negra, en la mezcla R (Figura 2b) explica la formación de fases cálcicas y la menor contracción de cocción de esta mezcla frente a la mayor contracción de la mezcla $\mathrm{V}$ (Figura 2 c), con un $50 \%$ de arcilla Roja (Tabla 1). El análisis mineralógico de la mezcla carbonatada $\mathrm{R}$ tratada a $900{ }^{\circ} \mathrm{C}$ and Grey clay significantly improves the behavior of the reference mixtures $R$ and $V$ during the drying process, as evidenced by the decrease of the linear shrinkage and Nosova index (Table 4). The use of Diatomaceous Earth causes a decrease in bulk density, mainly due to the high content of organic matter of this residue ( $L O I \approx 50$ wt.\%, Table 3). However, this decrease in density does not result in a loss of mechanical strength, which may be due in part to the plasticizing effect of the vegetable oil impregnated therein (27). The introduction in traditional mixtures ( $R$ and $V$ ) of Screen Glass causes an increase in density due to the greater density of the waste. Mechanical strengths of the unfired samples are above $35 \mathrm{~kg} / \mathrm{cm}^{2}$ in all cases and, therefore, a priori, all mixtures are suitable for use on an industrial scale, enabling manipulation of the unfired pieces for load in the furnace.

The behaviour of clays and mixtures during dynamic sintering tests is shown in Figure 2. It can be appreciated how the smectitic White clay, with high carbonate content, starts sintering before the illitic Yellow and Black clays (Fig. 2a). Due to the high content of carbonates in these three clays (Table 2), it is seen as crystalline calcium phases begin to form from 900-950 ${ }^{\circ} \mathrm{C}$ (Fig. 2a) providing a refractory nature in these materials (28). Conversely, the absence of carbonates in Red and Grey clays, and their melting nature (Fig. 2a), make them interesting materials for the production of clinker or stoneware products with higher added value. The higher content of Neogene clays, especially Yellow and Black, in mixture $R$ (Fig. $2 b$ ) explains the formation of calcium phases and the lower firing shrinkage in this mixture against a greater shringkage of mixture $V$ (Fig. 2c) with 50 wt.\% of Red clay (Table 1). Mineralogical analysis of reference carbonated $R$ mixture, fired at $900^{\circ} \mathrm{C}$, shows new calcium (anorthite and gehlenite, Table 5) in agreement 


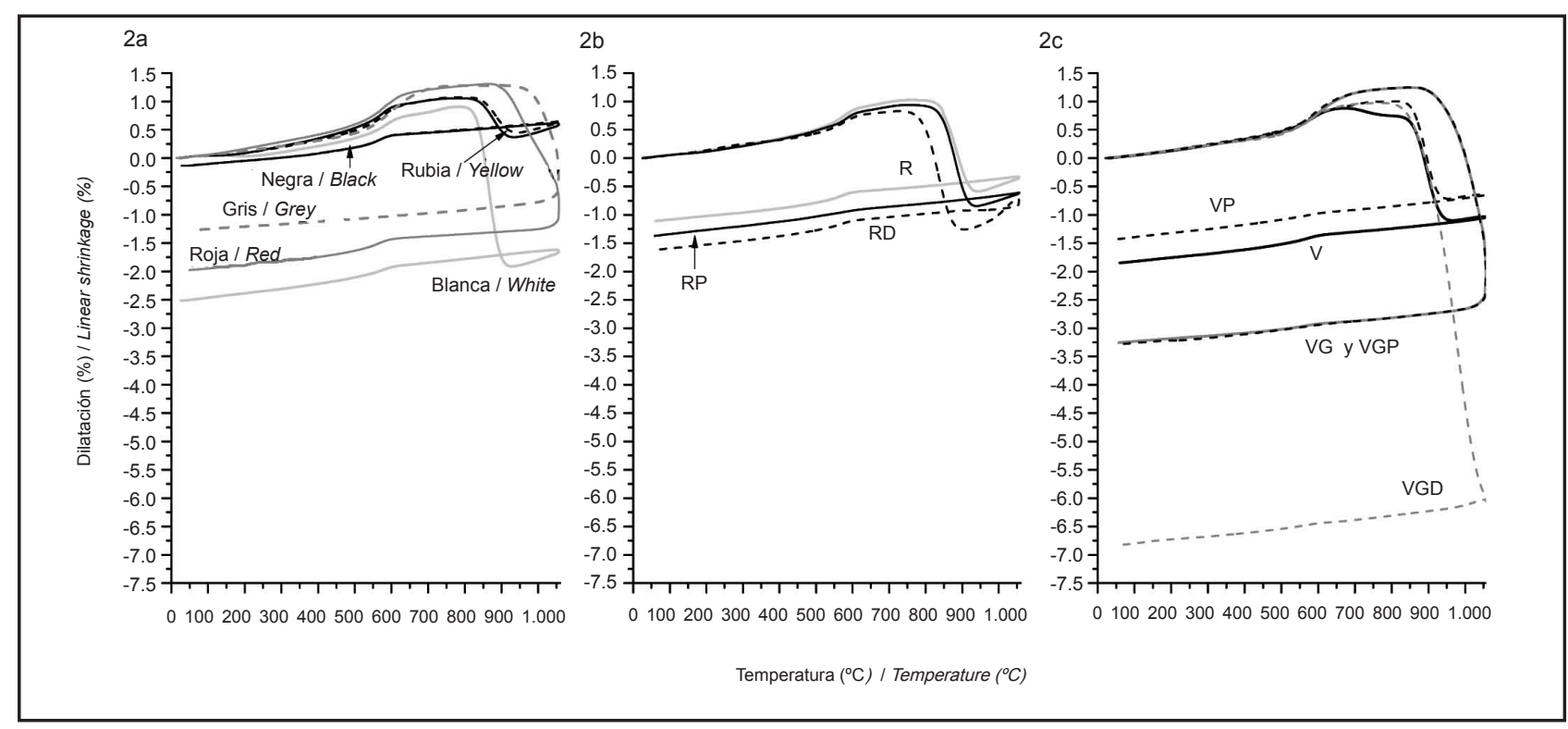

Figura 2. Análisis termodilatométricos de las arcillas y mezclas estudiadas: a) arcillas, b) materiales porosos (R), c) materiales densos $(\mathrm{V})$.

Figure 2. Dilatometric thermal analysis of individual clays and studied mixtures: a) clays, b) porous materials (R), c) dense materials $(\mathrm{V})$.

muestra la aparición de las nuevas fases cálcicas (anortita y gehlenita, Tabla 5), resultados que están de acuerdo con el amplio estudio de caracterización mineralógica de arcillas realizado en (29).

Las dilatometrías de las nuevas mezclas muestran que la utilización del Vidrio de Pantalla $(P)$ no modifica sustancialmente el comportamiento de las mezclas R, V y VG. La sustitución de las arcillas carbonatadas de la mezcla $V$ por la arcilla Gris en el material VG da lugar a una mayor fundencia (Figura 2c). Un mayor aumento de la contracción de cocción y, por lo tanto, de la fundencia, se observa al incorporar las Tierras Diatomeas en las mezclas R y VG, lo que puede ser debido al carácter amorfo y muy reactivo de estas partículas silíceas, produciendo, además, un descenso de la temperatura de comienzo de la sinterización de aproximadamente $100^{\circ} \mathrm{C}$. A esto hay que añadir que la combustión del gran contenido de materia orgánica de este residuo supone un aporte calorífico extra que puede contribuir a un ahorro en la cantidad de combustible necesaria en la zona de calentamiento de los hornos industriales. Por consiguiente, la utilización del residuo de Tierras Diatomeas puede suponer un ahorro energético importante en el proceso de cocción.

En la Figura 3, las curvas de gresificación muestran como las mezclas de arcillas con alto contenido en carbonatos ( $R, R D$ y $R P$ ) dan lugar a productos muy porosos con una importante capacidad de absorción de agua, que ronda o supera el $15 \%$ en peso de $\mathrm{H}_{2} \mathrm{O}$. La adición de Tierras Diatomeas (RD) produce un descenso de la densidad y de la resistencia mecánica y un importante aumento de la with the wide study on clay mineralogical charaterization performed in (29).

Dilatometric curves of the new mixtures show that the use of Screen Glass $(P)$ does not substantially modify the behavior of the mixtures $R, V$ and $V G$. The substitution of carbonated clays in mixture $V$ by Grey clay in the material VG results in a greater melting capacity thereof (Fig. 2c). A further increase in firing shrinkage and, hence, in melting capacity is observed by incorporating Diatomaceous Earth in mixtures $R$ and $V G$, which may be due to the amorphous nature of these very reactive siliceous particles, producing in addition, a decrease in temperature of onset of sintering of about $100{ }^{\circ} \mathrm{C}$. Besides, the combustion of the high organic matter content of this waste is an extra heat input, which may contribute to savings in the amount of fuel needed in the heating zone of industrial furnaces. Therefore, the use of Diatomaceous Earth waste can be a significant energy saving in the firing process.

In Figure 3, vitrification curves show how clay mixtures with high carbonate content $(R, R D$ and $R P)$ give rise to very porous products with a significant water absorption capacity, which is around or more than $15 w t . \% \mathrm{H}_{2} \mathrm{O}$. The addition of Diatomaceous Earth (RD) causes a decrease in bulk density and mechanical strength and a significant increase in the water absorption due to the additional 


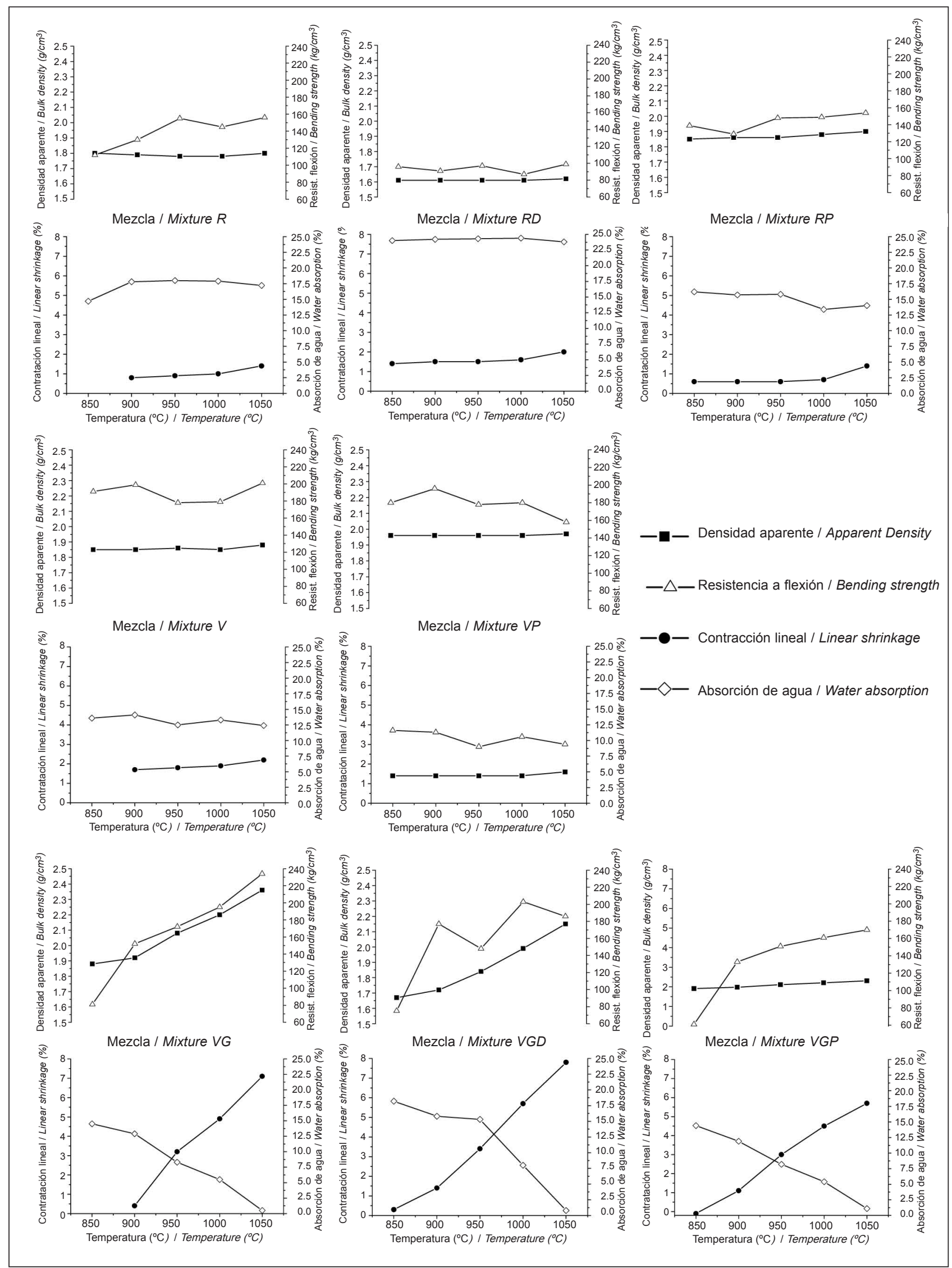

Figura 3. Diagramas de sinterización de las mezclas de arcillas estudiadas.

Figure 3. Sintering diagrams of the studied clay mixtures. 
Tabla 5 / Table 5

Análisis mineralógico (\% en peso) de la mezcla de arcillas carbonatadas $\mathrm{R}$ tratada a $900{ }^{\circ} \mathrm{C}$. Se ha utilizado el método Rietveld para la cuantificación de fases. Se aprecia la aparición de nuevas fases cálcicas (anortia y gehlenita).

Mineral analysis (wt.\%) of carbonated clays $R$ mixture fired at $900^{\circ} \mathrm{C}$. Rietveld method has beed used for phases quantification. It is noted the presence of new calcium phases (anortite and gehlenite).

\begin{tabular}{|c|c|}
\hline Fase / Phase & Mezcla / Mixture R $\mathbf{9 0 0}{ }^{\circ} \mathbf{C}$ \\
\hline Cuarzo / Quartz & $32.5 \pm 0.7$ \\
\hline Augita / Augite & $3.7 \pm 0.9$ \\
\hline Microclina / Microcline & $3.1 \pm 0.9$ \\
\hline Moscovita / Moscovite & $5.2 \pm 1.5$ \\
\hline Diópsido / Diopside & $7.2 \pm 1.8$ \\
\hline Anortita / Anorthite & $6.6 \pm 0.9$ \\
\hline Hematite / Hematite & $0.7^{*}$ \\
\hline Gehlenita / Gehlenite & $3.0 \pm 0.6$ \\
\hline Amorfo / Amorphous & $38.0 \pm 5.7$ \\
\hline
\end{tabular}

* Las fases con contenidos inferiores al $1 \%$ deben considerarse presentes como trazas / phase contents under $1 \mathrm{wt} \%$ must be considered as traces.

absorción de agua debido a la porosidad adicional formada por la combustión de la materia orgánica impregnada en este residuo. Este comportamiento refleja que el efecto producido por la porosidad formada en el material RD es superior al efecto fundente proporcionado por el propio residuo, como pone de manifiesto el comportamiento termodilatométrico (Figura 2b,c). Por el contrario, la adición del Vidrio de Pantalla (RP) produce ligeros aumentos de la densidad y resistencia mecánica y un descenso de la absorción de agua a partir de una temperatura de cocción de $950{ }^{\circ} \mathrm{C}$, debido al efecto fundente de dicho vidrio, lo que aumenta la vitrificación final.

Por lo que respecta a los materiales densos, al modificar la mezcla $V$ con arcilla Gris (VG) se produce un importante aumento del grado de vitrificación a las temperatura de trabajo habituales, $900-1050{ }^{\circ} \mathrm{C}$, otorgándole a dicho material una mayor densidad aparente, contracción lineal y resistencia mecánica junto con un gran descenso de la capacidad de absorción de agua, lo que le confiere la capacidad para poder fabricar productos gresificados a relativamente baja temperatura. La adición de Tierras Diatomeas y Vidrio de Pantalla sobre los materiales densos $(V P, V G D$ y $V G P)$ produce un efecto similar al descrito anteriormente para las mezclas $\mathrm{R}$.

Por lo tanto, se puede establecer, tal y como se observa en las predicciones empíricas de la Figura 1, que la adición de los residuos a las mezclas $R$ y $V$ en los porcentajes propuestos no produce un cambio significativo en el comportamiento de dicho material, que sigue presentando una porosidad importante, aunque se pueden mejorar aspectos como el secado o la resistencia mecánica y reducir la temperatura de cocción. En el caso de la mezcla $\mathrm{V}$, la nueva formulación con la arcilla Gris (VG) permite alcanzar una importante vitrificación del material, obteniéndose una absorción de agua por debajo del $2 \%$ en peso en los materiales sinterizados a $1.050^{\circ} \mathrm{C}$. Estos resultados se corresponden ampliamente con las predicciones previstas en la Figura 1. porosity formed by the combustion of organic material impregnated in this waste. This behavior reflects the effect of the porosity formed in the material $R D$ is greater than the effect provided by the melting capacity of the waste, as evidenced by the thermo-dilatometric behavior (Fig. 2b, c). By contrast, addition of Screen Glass (RP) produces slight increases in bulk density and mechanical strength and a decrease of water absorption values from a firing temperature of $950^{\circ} \mathrm{C}$, due to the melting effect of the glass, which increases the final vitrification in the material.

With respect to dense materials, changing the mixture $V$ with Grey clay (VG) produces a significant increase in the degree of vitrification at normal working temperature, 900-1050 ${ }^{\circ} \mathrm{C}$, giving the material a higher bulk density, linear shrinkage and mechanical strength along with a great decrease in water absorption capacity, which confers the ability to manufacture stoneware products at relatively low temperature. The addition of Diatomaceous Earth and Screen Glass on dense materials (VP, VGD and VGP) produces an effect similar to that described above for mixtures $R$.

Therefore, it can be established, as seen in the empirical predictions of Figure 1 , the addition of wastes to the mixtures $R$ and $V$ in the percentages given does not produce a significant change in the behavior of the material, that still has significant porosity, but it can improve aspects such as drying or mechanical strength and reduce the firing temperature. In the case of mixture $V$, the new formulation with Grey clay (VG) allows achieving a significant vitrification of the material, yielding a water absorption below 2 wt. \% in materials sintered at $1050^{\circ} \mathrm{C}$. These results largely correspond to the predictions provided in Figure 1. 


\subsection{Conductividad térmica}

En la Figura 4 se muestran los valores de conductividad térmica de una selección de los materiales obtenidos en función de la densidad. La adición a la mezcla porosa $R$ del residuo de Tierras Diatomeas $(\mathrm{RD})$ produce un descenso de la conductividad térmica debido a la disminución de la densidad aparente, mientras que el ligero aumento de dicha densidad asociado a la adición del Vidrio de Pantalla (RP) no se traduce en variaciones importantes de la conductividad térmica. Todas las mezclas porosas ( $R, R D, R P)$ presentan un carácter refractario, sin variaciones significativas de densidad aparente en el rango de temperaturas de cocción seleccionado, por lo que la conductividad térmica de estos materiales no varía significativamente en función de dicha temperatura. Por el contrario, los materiales con menor contenido de carbonatos (VG, VGP y, en menor medida, V) sufren una densificación a medida que aumenta la temperatura de cocción. Esto se debe a que la fase amorfa que se forma durante la sinterización va rellenando los huecos que forman los poros haciendo que la densidad aparente de la pieza aumente, lo que se manifiesta en un aumento de la conductividad térmica con la temperatura de cocción. Sin embargo, hay que destacar que el material VG sinterizado a $950{ }^{\circ} \mathrm{C}$, con una densidad aparente de $\approx 2,10 \mathrm{~g} / \mathrm{cm}^{3}$ y una absorción de agua inferior al $9 \%$ en peso, presenta una conductividad térmica $(\approx 0,83 \mathrm{~W} / \mathrm{mK})$ similar a la de los materiales $\mathrm{V}$ cocidos a $1.000-1.050{ }^{\circ} \mathrm{C}$, que poseen una porosidad significativamente superior $\left(\approx 1,86 \mathrm{~g} / \mathrm{cm}^{3}\right.$ de densidad aparente y una absorción de agua superior al $12 \%$ ). Este hecho se explica por la dependencia que la conductividad térmica tiene de la composición mineralógica y de la microestructura de los materiales (30). Así, se han descrito valores inferiores de conductividad térmica en materiales que contienen caolinita debido al tipo de

\subsection{Thermal conductivity}

Figure 4 shows thermal conductivity values of a selection of the obtained materials according to the density. The addition to the porous mixture $R$ of Diatomaceous Earth waste $(R D)$ causes a decrease in thermal conductivity due to the decrease in density, whereas the slight increase in density associated to the addition of Screen Glass (RP) is not resulting in substantial variations in thermal conductivity. All porous mixtures $(R$, $R D, R P$ ) have a refractory nature, without significant changes of density in the range of firing temperatures selected and thus thermal conductivity of these materials does not vary significantly with firing temperature. By contrast, materials with lower contents of carbonate (VG, VGP and to a lesser extent, $V$ ) have a densification with increasing firing temperature. This is because the amorphous phase formed during sintering will fill the voids which form pores, making the density of the pieces increase, which is manifested in an increase in thermal conductivity with the firing temperature. However, it is noteworthy that VG material sintered at $950{ }^{\circ} \mathrm{C}$, with a bulk density of $\approx 2.10 \mathrm{~g} / \mathrm{cm}^{3}$ and a water absorption less than 9 wt.\%, has a thermal conductivity $(\approx 0.83 \mathrm{~W} / \mathrm{mK}$ ) similar to $\mathrm{V}$ materials fired at $1000-1050{ }^{\circ} \mathrm{C}$, having a porosity significantly higher (density $\approx 1.86 \mathrm{~g} / \mathrm{cm}^{3}$ and water absorption over $12 w t . \%)$. This is explained by the dependence of the thermal conductivity on the mineralogical composition and microstructure of materials (30). In fact, lower conductivity values have been described for caolinite containing materials due to the kind of porosity developed by this phase during firing (30) and, thus, the higher content of kaolinite in the mixture VG may be responsible for levels of thermal conductivity similar to those in mixture $V$ with a higher porosity. However, a

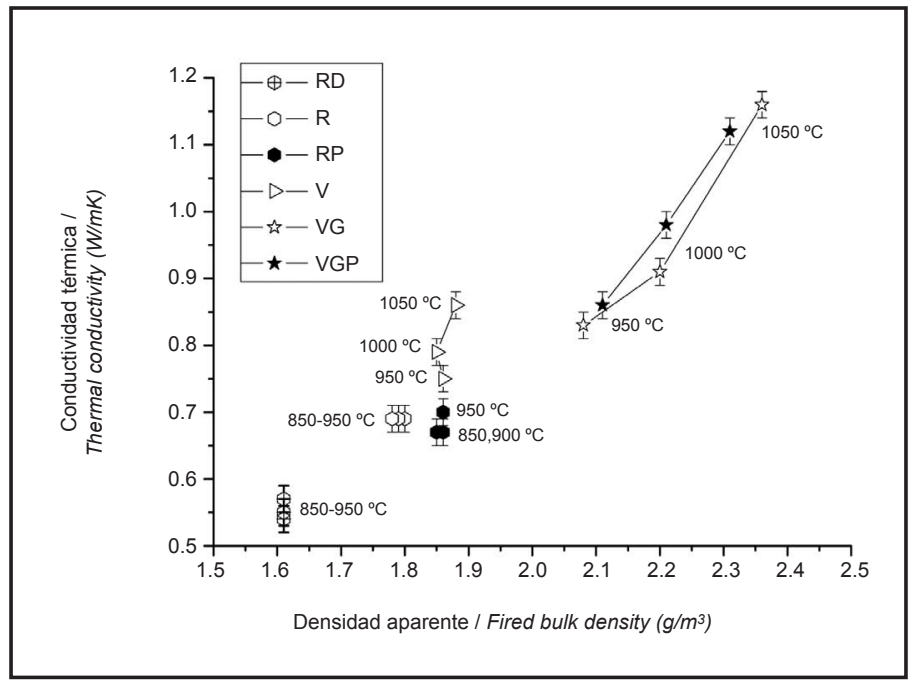

Figura 4. Valores de conductividad térmica en función de la densidad aparente. Se señalan también las temperaturas de cocción. Figure 4. Relation between thermal conductivity values and fired bulk density. Firing temperatures are also shown. 
porosidad asociado a las transformaciones de esta fase durante la cocción (30), por lo que el mayor contenido de caolinita presente en la mezcla VG puede ser responsable inicialmente de unos niveles de conductividad térmica similares a los de la mezcla $V$ con una mayor porosidad abierta, aunque un estudio microestructural, fuera del ámbito del presente trabajo, sería necesario para confirmar dicha hipótesis.

\section{CONCLUSIONES}

La adición de la arcilla Gris de Almuradiel y de los residuos industriales considerados: Tierras Diatomeas y Vidrio de Pantalla descontaminado, a las mezclas tradicionalmente utilizadas por el sector cerámico de Bailén, mantiene o mejora las propiedades tecnológicas, tanto en el comportamiento en seco como en cocido. Además, la utilización de residuos como materiales de partida conlleva un ahorro de materia prima y puede ayudar a mejorar el ciclo de vida de los productos cerámicos, disminuyendo así el impacto ambiental que genera su fabricación.

La utilización de Tierras Diatomeas en la mezcla porosa origina un descenso de la densidad, manteniendo una resistencia mecánica suficiente, y mejora notablemente la capacidad de aislamiento térmico de este material.

En materiales densos, la utilización del Vidrio de Pantalla descontaminado ayuda a la sinterización del material al formar más fase líquida, gracias al aporte de óxidos metálicos fundentes, por lo que disminuye la porosidad del material y la capacidad de absorción de agua.

El uso de la arcilla Gris mejora el comportamiento en secado y otorga una menor absorción de agua, propiedades con las que no contaban las mezclas de la industria ladrillera de Bailén, ofreciendo la posibilidad de fabricar productos gresificados, de mayor valor añadido, a las temperaturas de trabajo habituales de esta industria.

\section{AGRADECIMIENTOS}

La realización de estas actividades ha sido financiada por el Proyecto "Optimización y Mejora de las Propiedades Aislantes de los Materiales de Construcción de Arcilla Cocida" (PI44925, 2010), de la Consejería de Innovación, Ciencia y Empresa de la Junta de Andalucía, que ha sido cofinanciado por RedIndus: "Actuaciones de Reindustrialización en la provincia de Jaén" (Ministerio de Industria, Turismo y Comercio). Igualmente, se agradece la financiación previa del Proyecto: "Mejora en las características y usos de los productos cerámicos a partir de recursos existentes" (200500253, 2008), de la Consejería de Innovación, Ciencia y Empresa de la Junta de Andalucía. Las arcillas han microstructure study, out of the scope of the present work, would be necessary to confirm such hypothesis.

\section{CONCLUSIONS}

The addition of Grey clay from Almuradiel and the considered wastes: Diatomaceous Earths and Screen Glass, into mixtures traditionally used by the ceramic industry of Bailén, improves or keeps the technological properties in both drying and firing behavior. Furthermore, the use of waste as starting materials entails a saving of raw materials and may help to improve the life cycle of the ceramic products, thus reducing the environmental impact generated by their manufacture.

The use of Diatomaceous Earth in porous mixtures causes a decrease in density while maintaining a sufficient mechanical strength. It improves significantly the thermal insulation capacity of this material.

In dense materials, the use of Screen Glass helps to material sintering by forming additional liquid phase, thanks to the contribution of melting metal oxides, thereby decreasing material porosity and water absorption capacity.

The use of Grey clay improves drying and gives a lower water absorption capacity, properties that do not have the current mixtures used by the brick industry of Bailen, offering the ability to manufacture stoneware products, with higher value added, at operating temperatures typical of this industry.

\section{ACKNOWLEDGMENTS}

This work has been funded by the Project "Optimización y Mejora de las Propiedades Aislantes de los Materiales de Construcción de Arcilla Cocida" (PI44925, 2010), Consejería de Innovación, Ciencia y Empresa, Junta de Andalucía, that has been co-funded by RedIndus: "Actuaciones de Reindustrialización en la Provincia de Jaén" (Ministerio de Industria, Tuirsmo y Comercio). Likewise, previous finantial support of the Project: "Mejora en las características y usos de los productos cerámicos a partir de recursos existentes" (200500253, 2008), Consejería de Innovación, Ciencia y Empresa, Junta de Anadalucía, is also acknowledge. Clays have 
sido suministradas por Comercial Cerámica de Bailén, S.L, y Cerámica Malpesa, S.A, mientras que los residuos han sido facilitados por FCC-Ámbito. been provided by Comercial Cerámicas de Bailén S.L and Cerámica Malpesa S.A. Wastes have been provided by FCC-Ambito.

\section{BIBLIOGRAFÍA / BIBLIOGRPHY}

(1) González, I.; Galán, E.; Miras, A.; Aparicio, P.: "New uses for brick-making clay materials from the Bailén area (southern Spain)", Clay Minerals, vol. 33, no 3 (1998), pp. 453-465.

(2) Jiménez-Millán, J.; Molina, J.M.; Ruiz-Ortiz, P.A.: Advances in clay minerals, Proceedings of the Spanish-Italian Meeting on Clay Minerals, p. 230, Ortega, M.; Palomo, I.; López-Galindo, A. (Eds). Granada (1996).

(3) Alonso, A.; Álvarez, J.; Bueno, S.: I er Congreso Nacional de Minerales Industriales. Libro de Comunicaciones, p. 175, Fueyo Editores. Madrid (2010).

(4) Vázquez, M.; Jiménez-Millán, J.: "Materias primas ricas en arcilla de las Capas Rojas Triásicas (Norte de Jaén, España) para fabricar materiales cerámicos de construcción", Mater. Construcc.; vol. 54, no 273 (2004), pp. 5-20. http://dx.doi.org/10.3989/mc.2004. v54.i273

(5) Vázquez, M.; Jiménez-Millán, J.; Sánchez-Jiménez C.; Parras, J.: "Composición y propiedades cerámicas de pizarras de la Zona Centro Ibérica del Macizo Ibérico Meridional (Norte de Jaén, España)", Bol. Soc. Esp. Ceram. Vidrio, vol. 42, no 4 (2003), pp. 215-221.

(6) Vázquez, M.; Jiménez-Millán, J.: "Aplicabilidad de las margas de la Cordillera Bética (sur de la provincia de Jaén) para la fabricación de baldosas cerámicas prensadas", Mater. Construcc.; vol. 59, no 294 (2009), pp. 97-112. http://dx.doi.org/10.3989/mc.2009.v59.i294

(7) Sánchez Soto, P.J.; Pérez Rodríguez, J.L.: "Características generales, propiedades, yacimientos y aplicaciones de la pirofilita, II: Yacimientos, aplicaciones y utilización como materia prima cerámica", Bol. Soc. Esp. Ceram. Vidrio, vol. 37, no 5 (1998), pp. 359-368.

(8) LEED v3 §. Green Building Certification System, U. S. Green Building Council (2009).

(9) BREEAM ES ®. Método de de evaluación y certificación de la sostenibilidad de la edificación, BREEAM España (2012).

(10) Dondi, M.; Masigli, M.; Fabbri, B.: "Recycling of industrial and urban wastes in brick production - A review". Tile Brick Int.; vol. 13, no 3 (1997), pp. 218-225.

(11) Sánchez-Muñoz, L.; Carda Castelló, J.B.: Materias primas y aditivos cerámicos, p. 159, Ed. Faenza Editrice Iberica, S.L. Castellón (2002).

(12) Raut, S.P.; Ralegaonkar, R.V.; Mandavgane, S.A.: "Development of sustainable construction material using industrial and agricultural solid waste: A review of waste-create bricks", Construction and Building Materials, vol. 25, no 10 (2011), pp. 4037-4042. http://dx.doi. org/10.1016/j.conbuildmat.2011.04.038

(13) Ahorro energético en la industria de la cerámica estructural. Acción del Programa Thermie no I 227, Comisión Europea. Dirección General de la Energía (DGXVII) (1996).

(14) Demir, I.: "Effect of organic residues addition on the technological properties of clay bricks", Waste Management, vol. 28, no 3 (2008), pp. 622-627. http://dx.doi.org/10.1016/j.wasman.2007.03.019

(15) García-Ten, J.; Silva, G.; Cantavella, V.; Lorente, M.: "Utilización de materiales aligerantes en la fabricación de bloques de TermoarciIlaß. Influencia sobre la conductividad térmica y el comportamiento en el proceso", Conarquitectura, vol. 16, CA16 (2005), pp. 65-72.

(16) De la Casa, JA.; Lorite, M.; Jiménez, J.; Castro, E.: "Valorisation of wastewater from two-phase olive oil extraction in fired clay brick production", Journal of Hazardous Materials, vol. 169, no 1-3 (2009), pp. 271-278. http://dx.doi.org/10.1016/j.jhazmat.2009.03.095

(17) Monteiro, S.N.; Alexandre, J.; Margem, J.I.; Sánchez, R.; Vieira, C.M.F.: "Incorporation of sludge waste from water treatment plant into red ceramic", Construction and Building Materials, vol. 22, no 6 (2008), pp. 1281-1287. http://dx.doi.org/10.1016/j.conbuildmat.2007.01.013

(18) Martínez, M.L.; Eliche, D.; Cruz, N.; Corpas, F.A.: "Utilización de bagazo de la industria cervecera para la producción de ladrillos para la construcción", Mater. Construcc.; vol. 62, no 306 (2012), pp. 199-212. http://dx.doi.org/10.3989/mc.2012.v62.i306

(19) Andreola, F.; Barbieri, L.; Corradi, A.; Lancellotti, I.: "CRT glass state of the art. A case study: Recycling in ceramic glazes", J. Eur. Ceram. Soc.; vol. 27, no 2-3 (2007), pp. 1623-1629. http://dx.doi.org/10.1016/j.jeurceramsoc.2006.05.009

(20) Dondi, M.; Guarini, G.; Raimondo, M.; Zanelli, C.: "Recycling PC and TV waste glass in clay bricks and roof tiles", Waste Management, vol. 29, no 6 (2009), pp. 1945-1951. http://dx.doi.org/10.1016/j.wasman.2008.12.003

(21) Romero, M.; Rawlings, R.D.; Rincón, J.M.: "Development of a New Glass-Ceramic by Means of Controlled Vitrification and Crystallisation of Inorganic Wastes from Urban Incinirator", J. Eur. Ceram. Soc.; vol. 19, no 12 (1999), pp. 2049-2058. http://dx.doi.org/10.1016/ S0955-2219(99)00011-4

(22) Pelino, M.: "Recycling of zinc-hydrometallurgy wastes in glass and glass ceramics materials", Waste Management, vol. 20, no 7 (2000), pp. 561-568. http://dx.doi.org/10.1016/S0956-053X(00)00002-7

(23) Barabini, A.M.; Plescia, P.; Maccari, D.; Burragato, F.; Pelino, M.: "New materials from industrial and mining wastes: glass-ceramics and glass and rock-wool fibre", Int Jour. Mineral PROCESS, vol. 53, no 1-2 (1998), pp. 121-134. http://dx.doi.org/10.1016/S03017516(97)00062-8 
(24) Hernández-Crespo, M.S.; Rincón, J.M.: "Reciclado de Residuos de la Minería del Granito y de Incineradora de RSU en la Obtención de Nuevos Materiales Tipo Gres Porcelánico". Mater. Construcc.; vol. 50, no 260 (2000), pp. 49-62. http://dx.doi.org/10.3989/mc.2000. v50.i260

(25) Alonso-Santurde, R.; Andrés, A.; Viguri, J.R.; Raimondo, M.; Guarini, G.; Zanelli, C.; Dondi, M.: "Technological behaviour and recycling potential of spent foundry sands in clay bricks". J. Environ. Manage.; vol. 92, no 3 (2011), pp. 994-1002. http://dx.doi. org/10.1016/j.jenvman.2010.11.004

(26) Fiori, C.; Fabbri, B.; Donati, F.; Venturi, I.: "Mineralogical composition of the clay bodies used in the Italian tile industry", Appl. Clay Sci.; vol. 4, no 5-6 (1989), pp. 461-473. http://dx.doi.org/10.1016/0169-1317(89)90023-9

(27) Monteiro, S.N.; Vieira, C.M.F.: "Effect of oily waste addition to clay ceramic", Ceramics International, vol. 31, no 2 (2005), pp. 353358. http://dx.doi.org/10.1016/j.ceramint.2004.05.002

(28) Barba, A. y col.: Materias primas para la fabricación de soportes de baldosas cerámicas, p. 183, Instituto de Tecnología Cerámica. Castellón (2002).

(29) Fabbri, B.; Dondi, M.: Caratteristiche e difetti del laterizio, p. 71, Gruppo Editoriale Faenza Editrice S.p.A. Faenza (1995).

(30) García Ten, J.; Orts, M.J.; Saburit, A.; Silva, G.: "Thermal conductivity of traditional ceramics. Part II: Influence of mineralogical composition", Ceramics International, vol. 36, no 6 (2010), pp. 2017-2024. http://dx.doi.org/10.1016/j.ceramint.2010.05.013 